\title{
Parâmetros bioquímicos afetados pela alimentação segundo pesquisa estatística em análises clínicas no município de Araraquara, São Paulo, Brasil
}

\author{
Flávio Pereira Picheli ${ }^{1}$
}

Os problemas de saúde decorrentes do consumo de uma dieta qualitativamente inadequada são tão graves quanto a falta absoluta de acesso aos alimentos. A saúde alimentar e nutricional está ligada a qualidade econômica que ilustra quão possível é o acesso ao alimento seguro: equidade da fonte ao consumo, do preparo ao gerenciamento de resíduos e da qualidade de vida à promoção social. Objetivou-se o monitoramento de parâmetros bioquímicos de atendimentos do Sistema Único de Saúde pareado a indicadores socioeconômicos de Araraquara nos últimos cinco anos. Houve melhoria nos rendimentos e na renda per capita, no Índice de Desenvolvimento Humano, nas taxas de saneamento, alfabetização e expectativa de vida, além da diminuição da mortalidade infantil. Vê-se uma população crescente em consumo, formação e serviços, porém pré-disposta a doenças como diabetes e dislipidemias haja vista a maior frequência de classes intermediárias entre valores desejáveis e de alto risco clínico. Além disso, como são fatores ligados à alimentação, suas flutuações estão diretamente relacionadas aos hábitos individuais, havendo assim, a necessidade do estímulo da promoção da qualidade de vida pela alimentação saudável, atividades físicas e justiça social frente a menor dependência de medicamentos e produtos industrializados.

Palavras-chave: Segurança alimentar e nutricional, Análise clínica, Hábitos alimentares.

\section{Biochemical parameters affected by diet according to statistical research in clinical analysis in the city of Araraquara, Sao Paulo, Brazil.}

The health problems arising from consumption of a qualitatively in adequate diet are as serious as the ones arising from absolute lack of access to food. Food and nutritional health is linked to economic quality which shows how possible is the access to safe food: equity from the supply producer to the consumer, from food preparation to management of residues and from life quality to social promotion. The prupose of this study was to monitor the biochemical parameters of the Sistema Único de Saúde's (SUS) attendance paired with Araraquara's socioeconomic indicators in the last five years. There were improvements in both general and per capta income, in human development index, in the sanitation rates, literacy rates and life expectancy, there was also a decrease in the infant mortality rate. We can observe a population which is increasing in consumption, formation and services, but which is also pre-disposed to illnesses such as diabetes and dyslipidemia considering the higher frequency of intermediate classes between desired levels and high clinical risk. Besides, as these are factors linked to eating, their fluctuations are directly related to individual habits, so there is a stimulation need of the life quality promotion through eating healthly, physical activities and social justice against less medicine and manufactured goods dependence.

Key-words: Food and nutritional security, Clinical analysis, Feed habits.

\footnotetext{
${ }^{1}$ Especialista em controle de qualidade dos alimentos. Assistente de suporte acadêmico II.Correspondência: Departamento de Engenharia de Bioprocessos e Biotecnologia, Faculdade de Ciências Farmacêuticas de Araraquara (DBB-FCFAr-Unesp). Rodovia Araraquara - Jaú km 01, CEP 14801-902, Araraquara, São Paulo, Brasil. Fone: (16) 981.199.143.E-mail: flavio@fcfar.unesp.br
} 


\section{INTRODUÇÃO}

O crescente aumento das doenças crônicas, tanto em países desenvolvidos quanto em desenvolvimento, está relacionado às mudanças na dieta e no estilo de vida ocorrido nas últimas décadas,decorrente principalmente forte presença de produtos e serviços voltados às facilidades do dia a dia que impuseram tendenciosamente o sedentarismo, alimentos pouco nutritivos, consumismo sem necessidade real e a mídia apelativa. Uma frequência adequada de refeições, uma dieta equilibrada e a prática de atividades físicas moderadas são fatores importantes na promoção e manutenção da saúde ${ }^{[1]}$. Além disso, as características da dieta podem determinar não somente a saúde do indivíduo no momento presente, mas também, influenciar o desenvolvimento das Doenças Crônicas Não Transmissíveis como câncer, doenças cardiovasculares e diabetes em um período tardio.

Estas doenças possuem multiplicidade de fatores de risco com relação ou não às características etiológicas, epidemiológicas e genéticas da população, que uma vez sob estes diagnósticos, demandam por assistência continuada de serviços e ônus progressivo, na razão direta do envelhecimento dos indivíduos dessa população ${ }^{[1]}$ E como se observa em nível mundial, o Brasil também passa por uma revolução demográfica e nutricional, onde de modo amplo aumentaram a expectativa de vida e a proporção de idosos em relação aos demais grupos etários frente à queda dos índices de mortalidade e fecundidade. Sociedades modernas parecem convergir para uma dieta com elevada participação de gorduras saturadas e produtos refinados, baixas fontes de fibras e estilo de vida com reduzido interesse por atividade física que irão refletir diretamente sobre menores índices de desnutrição diante decrescente número de casos de sobrepeso, obesidade e doenças afins, ainda que uma menor taxa de desnutrição não signifique obrigatoriamente que todos tenham acesso a uma alimentação adequada, podendo mascarar o real estado de saúde do indivíduo.

Várias medidas bioquímicas têm sido propostas objetivando a detecção precoce de deficiências subclínicas e marginais, por isso a rotina clínica observa constantemente valores séricos de diversos analitos, além de contagens celulares como no hemograma. Pesquisas direcionadas à avaliação nutricional de pacientes e estudos populacionais têm consagrado a utilidade de alguns desses indicadores bioquímicos, bem como apontando a ineficácia de outros. Mesmo que se parta de uma análise ampla, sempre há necessidade de refino analítico e restrição dos exames no limiar de uma patologia estudada. Além das medidas básicas em bioquímica como colesterol, ureia e albumina, novos analitos surgiram como alternativas nos casos em que não se obtém sucesso no diagnóstico esperado, ou mesmo esses novos apresentam maior especificidade e sensibilidade; assim há modernização nos princípios e instrumentação analíticos aumentando frequências de trabalho e diminuindo limites de detecção frente a novos metabólitos determinados ${ }^{[2]}$. Dessa maneira, parece sensato afirmar que uma busca por parâmetros bioquímicos clássicos permitem delimitar novos testes para que se conclua um diagnóstico, bem como delinear comportamentos populacionais, haja vista a recorrência de alterações metabólicas nos quadros de patologias multifatoriais podendo culminar em estudos moleculares e citogenéticos.

Neste aspecto, o processo de acesso eficaz e amplo a uma alimentação proporcionalmente equilibrada e de origem confiável parece um problema potencialmente complexo numa sociedade pluralista e com discrepante perfil de distribuição de renda e poder de consumo. Se por um lado, proverse de uma alimentação rica em qualidade de nutrientes parece difícil e distante para muitos grupos sociais, por outro, comidas prontas congeladas, produtos de pouca riqueza nutricional, frituras, enlatados e à base de gorduras e açúcares simples parecem não corrigir o problema da fome e sim agravar quadros de carências nutricionais especificas e excesso de peso.

$\mathrm{O}$ ato de se alimentar instaura o conceito moderno de Segurança Alimentar e Nutricional, em que são abordados de forma holística visões de caráter social, econômico, ambiental, humano e nutricional propriamente dito. No Brasil, segundo a lei n⿳⺈⿴囗十一 1.346 de 15 de Setembro de 2006, que cria o Sistema Nacional de Segurança Alimentar e Nutricional, consiste em garantir a todos as condições de acesso aos alimentos básicos, seguros e de qualidade, em quantidade suficiente, de modo permanente e sem comprometer o acesso a outras necessidades essenciais, com base em práticas alimentares saudáveis, contribuindo para uma existência digna em um contexto de desenvolvimento integral da pessoa humana. Considerando o indivíduo autônomo nas 
suas decisões, a alimentação não pode perder sua condição de fonte de satisfação e realização, sendo saborosa e respeitando as práticas e hábitos alimentares das diferentes culturas. E na sua produção devem ser promovidas práticas diversificadas e integradas que garantam a relação harmônica do ser humano com a natureza. Uma alimentação que siga esses princípios é capaz de prevenir e reverter doenças carenciais e evitar as complicações das doenças degenerativas (anemias, obesidade, hipertensão). Ao mesmo tempo, seria capaz de estimular o fortalecimento de sistemas sustentáveis de produção de alimentos e gerar novos hábitos sociais de consumo, preparo e manutenção de vida saudável[3-5].

Reconhecido o valor da alimentação como fonte energética para realização das atividades diárias e o bom funcionamento do organismo, fica evidente a necessidade de se ter acesso a uma variedade de alimentos saudáveis, porque quando consumidos de maneira sobrepujante, contaminados ou com pouco valor nutricional podem levar a estados clínicos particulares (sobrepeso, dislipidemia, intoxicações), ou por sua vez, predisporem às condições endógenas e genéticas como nos quadros de alergias, autoimunidade, diabetes e complicações ${ }^{[1-6]}$. O acompanhamento de parâmetros nutri-funcionais parece ser uma ferramenta importante no equilíbrio do organismo e desta forma pode refletir em números a saúde coletiva de uma região. Neste panorama, o presente trabalho visa, por meio de estudos demográficos e estatísticos, traçar o perfil da população de Araraquara mapeando exames clínicos dos últimos cinco anos de um laboratório voltado ao atendimento do Sistema Único de Saúde (SUS), buscando relacionar a sociedade, a saúde coletiva e as características da alimentação dos munícipes promovendo uma reflexão sobre (in)segurança alimentar e nutrição local.

\section{Material e métodos}

Os dados analisados no presente trabalho foram obtidos junto ao Laboratório de Bioquímica Clínica e Urinálise do Laboratório Central do Núcleo de Atendimento à Comunidade da Coordenadoria de Análises Clínicas e Hemoterapia da Faculdade de Ciências Farmacêuticas da Universidade Estadual Paulista Júlio de Mesquita Filho (NAC/ CACH/ FCFAr - Unesp), localizado a Rua Expedicionários do Brasil, 1621, Centro, Araraquara, São Paulo, Brasil. Valores séricos de colesterol total e fração HDL, triglicerídeos, glicemia de jejum, sódio e potássio foram levantados do período correspondente aos 61 meses entre abril de 2009 e abril de 2014. Esse banco de dados englobou resultados de ambos os sexos e sem discriminação por faixa etária, compreendendo a toda entrada clínica do laboratório. O estudo absteve-se de autorização de comitês de ética por não violar a integridade social, nem física dos pacientes atendidos ao longo dos anos contabilizados, além de tomar dados retroativos segundo anonimato das fontes, importando somente o analito e o total dos valores mensais (5 anos). Além disso, foi adotada a Diretriz 9, das Diretrizes Éticas Internacionais para Pesquisa Biomédicas Envolvendo Seres Humanos, do Council for International Organization sof Medical Sciences - CIOMS, segundo o qual, para muitos tipos de pesquisas epidemiológicas o consentimento informado individual é impraticável ou desaconselhável[ ${ }^{[7]}$.

Os dados para cada parâmetro foram tabulados com auxílio do programa operacional do laboratório. Em posse dos dados brutos, foram postos em ordem crescente e determinados valores de máximo, mínimo, contagem total, média aritmética, desvio padrão e coeficiente de variação. Após análise crítica, foram agrupados respectivamente segundo os limites de referência adotados em literatura.A fim de se conhecer melhor a população atendida pelo laboratório, foram analisados dados de levantamentos estatísticos executados pela secretaria local do Instituto Brasileiro de Geografia e Estatística (IBGE). Foram analisados dados demográficos, natalidade, morbidade, infraestrutura, habitação saneamento básico e orçamento familiar, fornecidos pela agência da cidade. Todos esses dados são públicos e de livre acesso via site do Instituto, diretamente em informes periódicos da agência ou Diários Oficiais do Estado e da União.

\section{Resultados e discussão}

Fundada em 22 de agosto de 1817, Araraquara é uma das cidades de destaque do centro paulista ocupando uma região bastante ativa de circulação de pessoas e serviços. Há algum tempo foi condecorada com o maior valor do índice da Federação das Indústrias do Estado do Rio de Janeiro (FIRJAN) para qualidade de cidades atingindo a pontuação de 0,9359 em 2007. 
Vem mostrando evolução na qualidade global de seus munícipes (população estimada em 2015: 226.508 mil pessoas) ilustrado pelo valor crescente do Índice de Desenvolvimento Humano Municipal (IDH-M). Destacando-se na agroindústria da cana e da laranja por excelência, outros ramos estão movimentando a estrutura socioeconômica como o metalomecânico, a indústria têxtil, a tecnologia da informação e a aeronáutica. Perfil este que justifica bons índices demográficos e de qualidade de vida dos habitantes, como pode ser verificado na Tabela 1. Nesse sentido, e considerando o intervalo de estudo deste trabalho, o Censo de 2010 é muito expressivo e ilustra o processo de crescimento de forma geral do município ${ }^{[8-10]}$.

Tabela 1: Índices de qualidade socioeconômicos do município de Araraquara de acordo com o Censo demográfico oficial do IBGE em 2010.

\begin{tabular}{|c|c|}
\hline Indicador & Resultado $^{1}$ \\
\hline Mortalidade infantil (por mil) & 14,140 \\
\hline Expectativa de vida (anos) & 72,170 \\
\hline Índice de Desenvolvimento Humano Municipal (IDH-M) - Longevidade & 0,786 \\
\hline Taxa de Alfabetização (\%) & 94,80 \\
\hline Índice de Desenvolvimento Humano Municipal (IDH-M) - Educação & 0,915 \\
\hline Índice de Desenvolvimento Humano (IDH)* em 2010 & $* * 0,815$ \\
\hline Produto Interno Bruto (PIB) per capita (em milhões) & $24.842,520$ \\
\hline PIB - participação no estado (\%) & 0,390 \\
\hline Domicílios sem rendimento (\%) & 9,690 \\
\hline Domicílios com rendimento até $1 / 2$ salário mínimo (\%) & 1,020 \\
\hline Domicílios com rendimento até $1 / 4$ do salário mínimo (\%) & 4,210 \\
\hline Renda per capita $(\mathrm{R} \$)$ & 891,740 \\
\hline Abastecimento de água - nível de atendimento (\%) & 99,430 \\
\hline Coleta de lixo - nível de atendimento $(\%)$ & 99,960 \\
\hline Esgotamento sanitário - nível de atendimento (\%) & 98,890 \\
\hline \multicolumn{2}{|c|}{$\begin{array}{l}\text { *Em } 1991 \text { apresentava } 0,607 \text { (considerado baixo) e em } 2000,0,742 \text { (intermediário). } \\
\text { Valores acima de } 0,800 \text { são considerados altos. **Ocupava posição } 7 \text { no ranking do } \\
\text { Estado de São Paulo e } 14^{\circ} \text { nacional. }{ }^{1} \text { Valores segundo último Censo. }\end{array}$} \\
\hline
\end{tabular}

Fonte: IBGE-Cidades, IBGE, 2014.

Segundo a Fundação Sistema Estadual de Análise de Dados (SEADE), o IDH-M estando em 0,815 representa um valor considerado muito alto pelo Programa das Nações Unidas para o Desenvolvimento (PNUD), que o coloca numa escala positiva no contexto global do Estado. O IDH-M é um índice composto por três indicadores de desenvolvimento humano: vida longa e saudável (longevidade), acesso ao conhecimento (educação) e padrão de vida (renda). $O$ índice de educação evoluiu também de 0,411 em 1991 (baixo) para 0,782 (alto) em 2010.O percentual dos domicílios com renda per capita de até $1 / 2$ salário mínimo mostra a condição socioeconômica da população do município em comparação com a sua Região de Governo (RG), Região Administrativa (RA) e no Estado todo.Araraquara apresentou uma tendência de melhoria de renda para até $1 / 2$ salário mínimo entre 1991 e 2010, sendo que em 2000 foram registrados os menores índices em todas as localidades pesquisadas. No entanto, o percentual de domicílios sem declaração de renda aproximou-se de
10\% em 2010 e numa escalada crescente. Já a renda per capita aumentou significativamente de 2000 para 2010 (mais de 100\%) chegando a $\mathrm{R} \$ 891,74$, valor nominal acima dos outros locais pesquisados. Em relação aos serviços de abastecimento de água, coleta de lixo urbano e rede de coleta de esgoto, apresenta índice de atendimento superior ao do Estado de São Paulo e levemente superior aos da RA e RG, com aumento da cobertura gradual nos últimos anos. A disposição final do lixo se dá em aterro sanitário no município de Guatapará, $50 \mathrm{~km}$ de distância de Araraquara. $\mathrm{O}$ esgoto coletado $(99,96 \%)$ é tratado em $100 \%$ nas estações de tratamento de esgoto de Araraquara e Bueno de Andrada, segundo o Departamento Autônomo de Água e Esgoto (DAAE) ${ }^{[8-10]}$.

Em 2012, da parceria da Assembleia Legislativa do Estado de São Paulo (ALESP) com a Fundação SEADE surgiu um estudo do Índice Paulista de Responsabilidade Social (IPRS) que objetiva avaliar a qualidade de vida nos municípios 
paulistas e fornecer subsídios para os gestores na formulação de políticas públicas. O IPRS é um indicador inspirado no IDH e exprime sinteticamente um conjunto de dimensões para mensurar as condições de vida da população: riqueza, longevidade e escolaridade. Junto deste estudo, há outro levantamento de 2010, o chamado Índice Paulista de Vulnerabilidade Social (IPVS), segundo o qual é possível ter uma visão mais detalhada das condições de vida do município, com a identificação e a localização espacial das áreas que abrigam os segmentos populacionais mais vulneráveis à pobreza com base nos indicadores de renda, escolaridade e ciclo de vida familiar ${ }^{[11]}$.

Nas edições de 2008 e 2010 do IPRS, Araraquara classificou-se no Grupo 1, que engloba os municípios com bons indicadores de riqueza, longevidade e escolaridade. Neste período o consumo anual de energia elétrica por ligação no comércio, na agricultura e nos serviços variou de 16,6 MWh para 18,9 MWh e o consumo de energia elétrica por ligação residencial variou de 2,1 MWh para 2,2 MWh. O rendimento médio do emprego formal aumentou de $\mathrm{R} \$ 1.578$ para $\mathrm{R} \$ 1.634$ e o valor adicionado per capita cresceu de $\mathrm{R} \$ 14.782$ para $\mathrm{R} \$$ 15.710. Entretanto, seu índice está abaixo do nível médio estadual ocupando posição 89 no Estado. $\mathrm{Na}$ longevidade, a taxa de mortalidade infantil (por mil nascidos vivos) reduziu-se de 12,0 para 11,4; a taxa de mortalidade perinatal (por mil nascidos) aumentou de 14,2 para 15,3; a taxa de mortalidade das pessoas de 15 a 39 anos (por mil habitantes) diminuiu de 1,4 para 1,3; a taxa de mortalidade das pessoas de 60 a 69 anos (por mil habitantes) variou de 15,6 para 16,7 , o que ilustra um

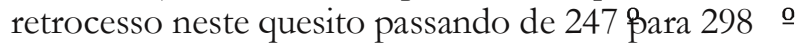
no Estado. Araraquara melhorou a pontuação de escolaridade no período ficando acima da média estadual. A despeito deste desempenho, o município perdeu posições no ranking dessa dimensão deixando a 56 posição para a 126 en 2010 , ainda que se verifique a taxa de atendimento escolar de crianças de 4 a 5 anos crescente de $93,3 \%$ para 100,0\%; a média da proporção de alunos da rede pública, que atingiram o nível adequado nas provas de português e matemática (5으 ano do ensino fundamental) aumentou de 42,3\% para 52,4\%; a média da proporção de alunos da rede pública, que atingiram o nível adequado nas provas de português e matemática ( 9 ano do ensino fundamental) variou de $23,6 \%$ para $23,8 \%$; o percentual de alunos com atraso escolar no ensino médio cresceu de $11,0 \%$ para 13,2\%. Complementando esta visão, a análise das condições de vida de seus habitantes mostra que a renda domiciliar média era de $\mathrm{R} \$ 2.696$, sendo que em 10,4\% dos domicílios não ultrapassava meio salário mínimo per capita. Em relação aos indicadores demográficos, a idade média dos chefes de domicílios era de 48 anos e aqueles com menos de 30 anos representavam 12,5\% do total. Dentre as mulheres responsáveis pelo domicílio 13,1\% tinham até 30 anos, e a parcela de crianças com menos de seis anos equivalia a $6,9 \%$ do total da população ${ }^{[11]}$.

$\mathrm{Na}$ tabela 2, são apresentadas as características dos grupos de vulnerabilidade do município de Araraquara.

Tabela 2: Indicadores levantados no Índice Paulista de Vulnerabilidade Social (IPVS) do município de Araraquara referentes ao ano de 2010.

\begin{tabular}{|c|c|c|c|c|c|c|}
\hline \multirow{2}{*}{ Parâmetro } & \multicolumn{6}{|c|}{ Grupo* } \\
\hline & $\mathrm{I}$ & II & III & IV & $\mathrm{V}$ & VII \\
\hline Vulnerabilidade & Baixíssima & $\begin{array}{l}\text { Muito } \\
\text { baixa }\end{array}$ & Baixa & $\begin{array}{c}\text { Média } \\
\text { (urbana) }\end{array}$ & $\begin{array}{c}\text { Alta } \\
\text { (urbana) }\end{array}$ & $\begin{array}{c}\text { Alta } \\
\text { (rural) }\end{array}$ \\
\hline Fração da população (\%) & 4,50 & 57,00 & 20,10 & 11,70 & 5,90 & 0,80 \\
\hline Rendimento nominal médio $(\mathrm{R} \$)$ & 5.512 & 2.921 & 2.207 & 1.643 & 1.412 & 1.154 \\
\hline Idade média do responsável pelo domicílio (anos) & 49 & 51 & 42 & 46 & 42 & 49 \\
\hline Responsáveis menores de 30 anos (\%) & 18,70 & 9,00 & 19,90 & 12,20 & 18,70 & 7,60 \\
\hline Crianças menores de 6 anos (\%) & 4,60 & 5,50 & 8,90 & 8,60 & 10,80 & 7,30 \\
\hline
\end{tabular}

*Araraquara não apresentou a classe VI, denominada Muito alta, no ano estudado.

Fonte: Fundação SEADE, 2014.

De modo amplo, o poder aquisitivo médio da no período de 2009 a 2012(Tabela $3)$. população de Araraquara sofreu sensível aumento 
Tabela 3: Algumas classes econômicas e seus rendimentos no município de Araraquara entre os anos 2009 e 2012, segundo a Fundação Sistema Estadual de Análise de Dados (SEADE) do Estado de São Paulo.

\begin{tabular}{|c|c|c|c|c|}
\hline Variável & 2009 & 2010 & 2011 & 2012 \\
\hline Despesas municipais com trabalho & 9.150 .940 & 11.887 .436 & 11.518 .654 & $*$ \\
\hline Rendimento médio do total de empregos formais & $1.522,16$ & $1.634,35$ & $1.750,34$ & $1.909,54$ \\
\hline $\begin{array}{l}\text { Rendimento médio dos empregos formais da Agricultura, } \\
\text { Pecuária, Produção Florestal, Pesca e Aquicultura }\end{array}$ & 892,80 & $1.136,10$ & $1.216,74$ & $1.292,76$ \\
\hline Rendimento médio dos empregos formais da Indústria & $1.731,63$ & $1.949,23$ & $2.057,37$ & $2.276,02$ \\
\hline Rendimento médio dos empregos formais da Construção & $1.155,87$ & $1.237,62$ & $1.477,05$ & $1.660,08$ \\
\hline $\begin{array}{l}\text { Rendimento médio dos empregos formais do Comércio } \\
\text { Atacadista e Varejista e do Comércio e Reparação de } \\
\text { Veículos Automotores e Motocicletas }\end{array}$ & $1.102,08$ & $1.166,88$ & $1.251,97$ & $1.395,11$ \\
\hline
\end{tabular}

*Não há valor mensurado para esta variável até o momento da realização do levantamento neste estudo.

Fonte: Fundação SEADE, 2014.

Os valores dos anos subsequentes (e integrantes deste trabalho) não se encontram nas bases de dados do IBGE e da Fundação SEADE. Porém, o que se verifica é aumento dos rendimentos em diversos setores econômicos. O rendimento costuma ser empregado, sobretudo para gastos de moradia e alimentação, por isso, numa suposição, houve melhoria no poder de compra destes bens pela população. As classes econômicas de valor primário como agricultura e pecuária e a classe da indústria mostram aumentos mais intensos quando comparado ao setor de serviços. Observadas algumas evidências da ascensão de Araraquara quanto à qualidade de vida, pode-se então associar ao que vem ocorrendo no país, onde há expressivo aumento da renda familiar que leva a maior demanda de bens para subsistência e destes, destacase a alimentação. Ter acesso a uma dieta essencialmente rica em nutrientes tem se tomado foco constante de boa parte da população que vem se preocupando com o controle de peso, consumo de produtos integrais, estímulo de comércios e produtores locais, bem como produtos orgânicos e certificados. Por outro lado, em situações opostas, encontramos pessoas tendo acesso a produtos industrializados, refinados, com excesso de aditivos, ou seja, limitações dietéticas geralmente impostos pela rotina de vida estressante e tumultuada. Neste caso, o indivíduo começa a valer-se de uma alimentação inadequada, que por sua vez, corrobora para vir à tona processos metabólicos talvez naturalmente ativos apenas em longo prazo, mas que tem sua predisposição aumentada pelo excesso de estímulos exógenos que poderão ser traduzidos em excesso de peso, diabetes, dislipidemias e hipertensão arterial $[1-3,6]$.

Consoante à prática de alimentar-se, está a realidade de quão adequado a é esse acesso ao alimento. Idealmente a alimentação deve fornecer calorias, proteínas, vitaminas, lipídios, carboidratos, fibras e minerais necessários à boa qualidade de vida de forma segura e balanceada. $\mathrm{O}$ indivíduo deve reconhecer a qualidade do alimento que escolhe e cobrar informações dos produtores por meio de rotulagem clara e completa. Nem sempre essas informações são compreensíveis e as informações nutricionais mais complicam que elucidam no momento da escolha do alimento. Hoje muitas informações são extraídas de experiências norteamericanas que não necessariamente correspondem à realidade brasileira, aos hábitos locais, à tradição nacional e ao próprio contexto geográfico do país. As associações entre alimentação, nutrição e saúde foram sendo construídas gradativamente de acordo com o progresso das civilizações, onde com o decorrer do tempo, as bases da ciência nutricional moderna demonstraram a existência da relação entre certas doenças já conhecidas e o consumo habitual de alimentos carentes ou ricos em nutrientes. Essa evolução culminou com o surgimento da Epidemiologia Nutricional, que procura descrever a distribuição e a magnitude das doenças relacionadas com a alimentação e a nutrição e aos desequilíbrios nutricionais e alimentares da população, assim como elucidar causas da enfermidade e proporcionar informações necessárias para o planejamento de ações de saúde destinadas a prevenir, controlar e tratar as doenças descobertas, o que a torna também uma ferramenta de saúde coletiva ${ }^{[3-5,2]}$.

Partindo do contexto de que a alimentação apresenta relação direta com as Doenças Crônicas Não Transmissíveis, a avaliação alimentar é indispensável para correlação dos estudos epidemiológicos com práticas concretas de 
segurança alimentar e melhoria da qualidade de vida. A avaliação da alimentação é complexa e muitas vezes difícil. A ingestão ou o real consumo do indivíduo ou de uma coletividade não será igual ao seu consumo habitual e suas diferenças derivam da maneira como os métodos colhem e mensuram a informação alimentar e nutricional. Desta forma há diferenças sutis entre alimentação habitual e atual, em que a primeira é a média do consumo de alimentos em um longo período de tempo mantendo um padrão alimentar, e a segunda é a média do consumo alimentar de um curto período de tempo corrente. Isto fez elaborar formas de abordagem no conhecimento do estado de alimentação do indivíduo: informação direta de consumo calculando a ingestão de nutrientes, medida de composição ou dimensão corporal que refletem a alimentação prolongada e, por fim, medidas bioquímicas de sangue e tecidos que constroem indicadores da alimentação e aliado a este estão os dados correntes, segundo os quais a melhor forma de estudo de um grupo ou parâmetro, se dá pelo acompanhamento instantâneo da evolução do mesmo ${ }^{[13]}$.

Aceitando que a população se transforma constantemente, tanto em quantidade de indivíduos, como distribuição em diferentes nichos sociais e ambientais, o processo de coleta e análise de dados paralelamente ao progresso ou regresso contribui para explicar a própria prosperidade ou falências do coletivo. Mas como monitorar a saúde dessa população? Os dados correntes na análise clínica podem ser uma das respostas a esse inquérito multidisciplinar. As visitas aos médicos estão cada vez mais combinadas aos estudos nutricionais. Podese dizer que o estado nutricional é um componente real da condição de saúde de um indivíduo e é influenciado pelo consumo e utilização de nutrientes e pelas necessidades individuais, sendo identificado pela correlação de informações obtidas da avaliação antropométrica e dietética, exames físicos e dados bioquímicos. A alteração do estado nutricional por deficiência ou excesso de ingestão está intimamente ligada com o aparecimento e agravo de doenças ${ }^{[0]}$. Não apenas as carências nutricionais estão associadasàs complicações e alterações metabólicas, mas também aos quadros de ingestão alimentar excessiva e/ou inadequada. Desta forma, inquéritos alimentares somam-se aos marcadores bioquímicos a fim de se ter um diagnóstico holístico de um paciente. Alguns marcadores são capazes de fornecer quantidades absolutas de um nutriente, enquanto outros medem sua concentração em plasma, soro, urina e tecidos. Sua vantagem essencial é justamente maior precisão e confiabilidade frente a técnicas subjetivas isoladas. A concentração biológica de um atributo bioquímico não depende somente da fisiologia e da ingestão, mas também da herança genética, atividades físicas e tabagismo. Garantida uma coleta e manipulação adequadas, seus valores devidamente interpretados, podem representar o estado real do indivíduo e nortear diagnósticos mais precisos e confiáveis ${ }^{[1,2,6]}$.

Neste quadro surge uma questão complementar que diz respeito ao acesso a essas técnicas de estudo clínico. Muitos estratos da população podem recorrer aos chamados Planos de Saúde, sejam de forma particular ou cooperada. Outras precisam de acesso subsidiado pela esfera pública por meio do SUS. Embora o número de beneficiários de planos de saúde continue a crescer em Araraquara, isso não é restritamente uma garantia de sucesso. Segundo a Agência Nacional de Saúde Suplementar (ANS) em agosto de 2013 eram 108.805 usuários, passando para 110.311 em janeiro de 2014. Se adotarmos a projeção populacional estimada pelo IBGE (226.508 pessoas em 2015), isto corresponde a aproximadamente metade da população $(48,70 \%)$. E o setor tende a expandir, uma vez que a população busca cada vez mais atendimento particulartendo em vista a insatisfação do serviço público ainda que as operadoras de planos de saúde ocupem os primeiros lugares nos rankings de insatisfação dos consumidores ${ }^{[14]}$. Assim, há um quadro paradoxal na saúde, em que a população de baixa renda se encontra dependente do serviço prestado pela gestão pública em contrapartida aos consumidores particulares. Estes se veem obrigados a se sujeitarem a serviços limitados para fazer valer o direito à saúde e bemestar, ou seja, não há diferença concreta na qualidade da acessibilidade do cidadão ao serviço médico.Isso se torna mais importante quando observada a taxa geométrica de crescimento da população que passou de 1,35\% em 2010 para 1,05\% em 2014, ou seja, redução de aproximadamente $22,23 \%$, seguido do aumento da taxa do índice de envelhecimento passando de 72,83\% em 2009 para 88,51\% em 2014. Este ilustra a proporção de pessoas de 60 anos e mais por 100 indivíduos de 0 a 14 anos, que mostra um aumento da população idosa diante dos jovens ${ }^{[?]}$.

Outro ponto de discussão é o que se despende à saúde. Muitas doenças promovem onerosos gastos ao curso de sua evolução, e parte dessas despesas são direcionadas aos cofres públicos. Muitas parcelas da população dependem diretamente de produtos e serviços fornecidos por 
programas sociais e unidades básicas de saúde. Não há leitos médicos e de internação para todos, além de atendimentos emergenciais ou urgentes dependerem de plantões médicos saturados, poucas especialidades disponíveis e tempo de espera considerável[ ${ }^{[0]}$ As cifras investidas nessas subvenções ligadas à saúde coletiva são crescentes, mas não acompanhadas de aumentos consideráveis de unidades e leitos de atendimento como se verifica na Tabela 4.

Tabela 4: Indicadores de gastos em saúde do município de Araraquara entre os anos 2009 e 2013 segundo a Fundação Sistema Estadual de Análise de Dados (SEADE) do Estado de São Paulo.

\begin{tabular}{|c|c|c|c|c|c|}
\hline Variável & 2009 & 2010 & 2011 & 2012 & 2013 \\
\hline Total de despesas em saúde $(\mathrm{R} \$)$ & 138.011.046 & 151.726 .146 & 165.336 .231 & $*$ & $* 1$ \\
\hline Gastos em atenção básica $(\mathrm{R} \$)$ & 68.719 .645 & 81.023 .903 & 78.713 .300 & * & $*$ \\
\hline Gastos em assistência hospitalar e ambulatorial (R\$) & 53.492 .035 & 52.961 .588 & 66.433 .950 & * & $*$ \\
\hline Gastos em suporte profilático e terapêutico $(\mathrm{R} \$)$ & $*$ & $*$ & $*$ & $*$ & $*$ \\
\hline Gastos em vigilância sanitária $(\mathrm{R} \$)$ & 1.448 .138 & 1.526 .676 & 1.702 .963 & $*$ & $*$ \\
\hline Gastos em vigilância epidemiológica $(\mathrm{R} \$)$ & 4.638 .582 & 4.618 .125 & 6.565 .560 & $*$ & $*$ \\
\hline Gastos em alimentação e nutrição ( $\mathrm{R} \$)$ & 38.080 & $*$ & $*$ & $*$ & $*$ \\
\hline Unidades de atenção básica & 28 & 28 & 30 & 30 & 31 \\
\hline Leitos de Internação & 543 & 504 & 545 & 587 & 586 \\
\hline Leitos do Sistema Único de Saúde (SUS) & 238 & 238 & 238 & 278 & 268 \\
\hline
\end{tabular}

${ }^{1}$ As lacunas preenchidas pelo símbolo * significam valores ainda não mensurados para a variável apresentada na tabela até o momento da coleta de dados deste estudo.

Fonte: Fundação SEADE, 2014.

Os dados coletados neste trabalho foram agrupados consoantes às classes de risco recomendadas pela Sociedade Brasileira de Cardiologia (SBC) ${ }^{[15]}$ : para o colesterol total serão considerados desejáveis valores menores que 200mg.dL $\mathrm{mL}^{-1}$; limítrofes, valores maiores que 200 $\mathrm{mg} \cdot \mathrm{dL}^{-1} \mathrm{e}$ menores que $239 \mathrm{mg} \cdot \mathrm{dL}^{-1}$, e finalmente altos, valores iguais ou maiores que $240 \mathrm{mg} \cdot \mathrm{dL}^{-1}$. Para a fração HDL, são desejáveis os valores maiores que $60 \mathrm{mg}$. $\mathrm{dL}^{-1} \mathrm{e}$ baixos os inferiores a 40 mg.dL ${ }^{-1}$ não havendo classificação para o intervalo entre esses valores. Para os triglicerídeos, serão considerados desejáveis valores menores que 150mg.dL ${ }^{-1}$;limítrofes, menores que $200 \mathrm{mg} \cdot \mathrm{dL}^{-1} \mathrm{e}$ maiores que $150 \mathrm{mg} . \mathrm{dL}^{-1}$; altos, aqueles acima de 200 mg.dL ${ }^{-1}$ e menores que 499 mg.dL ${ }^{-1}$ e por fim, muito altos, os que ultrapassarem $499 \mathrm{mg} \cdot \mathrm{dL}^{-1}$. Foi adotada também a recomendação da Sociedade Brasileira de Diabetes (SBD) ${ }^{[16]}$ : para glicemia de jejum, serão ditos normais valores inferiores a $100 \mathrm{mg} \cdot \mathrm{dL}^{-1}$; sujeitos à tolerância diminuída a glicose, os situados entre 100 e $126 \mathrm{mg} \cdot \mathrm{dL}^{-1} \mathrm{e}$ por fim, diabetes mellitus, os iguais ou acima de $126 \mathrm{mg} . \mathrm{dL}^{-1}$. Para eletrólitos são adotados os intervalos de 138 a $148 \mathrm{mEq} \cdot \mathrm{L}^{-1}$ para sódio e 3,8 a $5,5 \mathrm{mEq} \cdot \mathrm{L}^{-1}$ para potássio ${ }^{[1-20]}$. Vale ressaltar que o jejum é definido para SBD como a falta de ingestão calórica por no mínimo de 8h. Além disso, segundo a SBC as determinações do colesterol total e HDL podem ser feitas sem jejum prévio ao passo que a determinação de triglicerídeos deve ser feita com jejum de $12 \mathrm{~h}^{[15,16]}$.

O total de atendimentos coletados e analisados neste estudo pode ser visto na Tabela 5 . 
Parâmetros bioquímicos, analises clínicas e alimentação. Picheli.

Tabela 5: Classificação dos exames obtidos do levantamento realizado no laboratório de análises clínicas da Faculdade de Ciências Farmacêuticas de Araraquara segundo recomendação da Sociedade Brasileira de Cardiologia $^{[15]}$ e da Sociedade Brasileira de Diabetes ${ }^{[16]}$ entre os anos de 2009 e 2014.

\begin{tabular}{|c|c|c|c|c|c|c|c|c|}
\hline \multirow{2}{*}{ Parâmetro } & \multirow{2}{*}{$\begin{array}{l}\text { Classe } \\
\text { de risco }\end{array}$} & \multirow{2}{*}{ Frequência } & \multicolumn{5}{|c|}{ Período* } & \multirow{2}{*}{$\begin{array}{c}\text { Total } \\
\text { (classe) }\end{array}$} \\
\hline & & & $2009 / 10$ & $2010 / 11$ & $2011 / 2012$ & $2012 / 13$ & $2013 / 14$ & \\
\hline \multirow{7}{*}{$\begin{array}{c}\text { Colesterol } \\
\mathrm{n}=131329 \\
\left(18,51 \% 0^{3}\right)\end{array}$} & \multirow{2}{*}{ Alto } & $\mathrm{n}$ & 2922 & 3697 & 3787 & 5222 & 5137 & 20765 \\
\hline & & $\% 1$ & 16,85 & 15,74 & 13,47 & 16,43 & 16,79 & \\
\hline & \multirow{2}{*}{ Limitrofe } & $\mathrm{n}$ & 4578 & 6512 & 7489 & 9307 & 8805 & 36691 \\
\hline & & $\%$ & 26,40 & 27,73 & 26,63 & 29,2765 & 28,7811 & \\
\hline & \multirow{3}{*}{ Desejável } & $\mathrm{n}$ & 9843 & 13276 & 16842 & 17261 & 16651 & 57456 \\
\hline & & $\%$ & 56,76 & 56,53 & 59,90 & 54,30 & 54,43 & \\
\hline & & Total & 17343 & 23485 & 28118 & 31790 & 30593 & 131329 \\
\hline \multirow{7}{*}{$\begin{array}{c}\text { HDL } \\
\mathrm{n}=97736 \\
(13,78 \%)\end{array}$} & \multirow{2}{*}{ Desejável } & $\mathrm{n}$ & 1028 & 2098 & 2787 & 3417 & 3593 & 12923 \\
\hline & & $\%$ & 8,69 & 12,66 & 13,83 & 14,12 & 14,39 & \\
\hline & \multirow{2}{*}{ Transiçãao ${ }^{2}$} & $\mathrm{n}$ & 6997 & 9610 & 11878 & 14029 & 14293 & 56807 \\
\hline & & $\%$ & 59,13 & 57,99 & 58,95 & 57,96 & 57,22 & \\
\hline & \multirow{3}{*}{ Baixo } & $\mathrm{n}$ & 3809 & 4863 & 5485 & 6757 & 7092 & 28006 \\
\hline & & $\%$ & 32,19 & 29,35 & 27,22 & 27,92 & 28,39 & \\
\hline & & Total & 11834 & 16571 & 20150 & 24203 & 24978 & 97736 \\
\hline \multirow{9}{*}{$\begin{array}{c}\text { Triglicerídeos } \\
\mathrm{n}=133216 \\
(18,79 \%)\end{array}$} & \multirow{2}{*}{$\begin{array}{l}\text { Muito } \\
\text { alto }\end{array}$} & $\mathrm{n}$ & 326 & 471 & 590 & 634 & 593 & 2614 \\
\hline & & $\%$ & 1,84 & 2,03 & 2,12 & 1,94 & 1,86 & \\
\hline & \multirow{2}{*}{ Limitrofe } & $\mathrm{n}$ & 2968 & 3966 & 4799 & 5882 & 5811 & 23426 \\
\hline & & $\%$ & 16,73 & 17,11 & 17,27 & 18,02 & 18,24 & \\
\hline & \multirow{2}{*}{ Alto } & $\mathrm{n}$ & 3437 & 4870 & 5968 & 7149 & 6756 & 28180 \\
\hline & & $\%$ & 19,37 & 21,01 & 21,47 & 21,90 & 21,21 & \\
\hline & \multirow{3}{*}{ Desejável } & $\mathrm{n}$ & 11009 & 13869 & 16437 & 18985 & 18696 & 78996 \\
\hline & & $\%$ & 62,06 & 59,84 & 59,14 & 58,15 & 58,69 & \\
\hline & & Total & 17740 & 23176 & 27794 & 32650 & 31856 & 133216 \\
\hline \multirow{7}{*}{$\begin{array}{c}\text { Glicemia } \\
\text { de jejum** } \\
\mathrm{n}=201127 \\
(28,35 \%)\end{array}$} & \multirow{2}{*}{$\begin{array}{l}\text { Diabetes } \\
\text { mellitus }\end{array}$} & $\mathrm{n}$ & 4929 & 6591 & 8220 & 9810 & 8432 & 37982 \\
\hline & & $\%$ & 17,20 & 18,09 & 19,16 & 20,36 & 18,76 & \\
\hline & \multirow{2}{*}{$\begin{array}{l}\text { Tolerância } \\
\text { diminuida }\end{array}$} & $\mathrm{n}$ & 4587 & 7010 & 8655 & 12167 & 10190 & 42609 \\
\hline & & $\%$ & 16,01 & 19,24 & 20,18 & 25,26 & 22,67 & \\
\hline & \multirow{3}{*}{ Normal } & $\mathrm{n}$ & 19144 & 22838 & 26025 & 26200 & 26329 & 120536 \\
\hline & & $\%$ & 66,80 & 62,68 & 60,66 & 54,38 & 58,57 & \\
\hline & & Total & 28660 & 36439 & 42900 & 48177 & 44951 & 201127 \\
\hline \multirow{2}{*}{\multicolumn{2}{|c|}{$\begin{array}{c}\text { Sódio } \\
\mathrm{n}=55112 \\
(7,77 \%) \\
\end{array}$}} & $\mathrm{n}$ & 2595 & 7268 & 14545 & 15458 & 15246 & 55112 \\
\hline & & $\%$ & 4,71 & 13,19 & 26,39 & 28,05 & 27,66 & \\
\hline \multirow{2}{*}{\multicolumn{2}{|c|}{$\begin{array}{c}\text { Potássio } \\
\mathrm{n}=89760 \\
(12,65 \%)\end{array}$}} & $\mathrm{n}$ & 7969 & 13150 & 20699 & 23739 & 24203 & 89760 \\
\hline & & $\%$ & 8,88 & 14,65 & 23,06 & 26,45 & 26,96 & \\
\hline
\end{tabular}

Total de exames pesquisados: $\mathrm{n}=709405$

*Contagem de abril a maio do ano seguinte. Estudo iniciado em abril/09 porque corresponde ao ano a partir do qual todos os exames realizados no laboratório clínicos eram contabilizados digitalmente. **Dados coletados apenas da glicemia de jejum que exclui as modalidades pós-sobrecarga, curvas glicêmicas e pósprandial. ${ }^{1}$ Percentuais em relação ao total de exames do parâmetro no intervalo anual de coleta de dados. ${ }^{2} \mathrm{~A}$ fração HDL situada entre valores menores que $60 \mathrm{mg}^{\mathrm{dL}} \mathrm{L}^{-1} \mathrm{e}$ acima de $40 \mathrm{mg} \cdot \mathrm{dL}^{-1}$ não possui denominação oficial. ${ }^{3}$ Percentual em relação ao total de exames dos 61 meses.

Fonte: NAC-CACH-FCFAr/ Unesp, 2014. 
Por meio do banco de dados do laboratório clínico, foi possível selecionar, a cada ano de interesse, a frequência de cada um dos parâmetros bioquímicos dentre os 709.405 exames executados nos 61 meses estudados, para então dividi-los nas classes preconizadas pela SBC e pela SBD. Por exemplo, no mês de abril de 2009, foram realizados 737 exames de colesterol total dos quais 348 se encontravam com valores desejáveis, 222, limítrofes e 167 altos. Tal cálculo procedeu-se para os outros meses, tipos de exames e classes de risco. De maneira global, todo parâmetro bioquímico analisado apresentou crescimento de frequência ao longo dos anos estudados. No último intervalo de estudo (período de maio 2013 a abril de 2014), os parâmetros apresentam uma sutil diminuição, justificada pelo menor número de atendimentos realizados, de acordo com o sistema de distribuição regional de cotas do SUS, que devem ser estabelecidas e cumpridas no período de fatura mensal. Assim, não havendo cota extra, cessa-se a fatura e os atendimentos são suspensos. Passado o período de cálculo, nova fatura é iniciada e o procedimento, restabelecido.

Considerados doseamentos diretamente influentes nos quadros de diagnósticos primários de diabetes e dislipidemias, os exames de glicemia de jejum etriglicérideos,que juntos correspondem a aproximadamente $47,13 \%$ dos exames analisados (n $=334.343$ ), são os mais requisitados pelos médicos nos atendimentos do sistema SUS. O atributo colesterol total também aparece em quantidade semelhante aos triglicerídeos, fato este recorrente na prática clínica do lipidograma, ainda que o regimento SUS recomende a descrição isolada de cada fração colesterol e triglicerídeo. As medidas de sódio $(7,77 \% ; \mathrm{n}=55112)$ e potássio $(12,65 \% ; \mathrm{n}=$ 89760) séricos são menos expressivas diante dos demais, exames no período dos 61 meses. Aquelas são recorrentes aos pacientes com provável histórico cardíaco ou nefrológico, portanto, não são considerados exames da rotina clínica, porém ainda sim relacionados ao consumo de industrializados e aditivos alimentares.

Analisando cada parâmetro, inicialmente pode-se observar que há maior fração da população na classe desejável do colesterol (valores superiores a $50 \%$ em todos os anos estudados. Ou seja, dos exames de colesterol total realizados ano a ano, os valores observados são menores que $200 \mathrm{mg} \cdot \mathrm{dL}^{-1}$. De qualquer forma, a fração limítrofe também é expressiva a cada ano e isto deve ser levado em conta, representando atenção à saúde devido aos valores que ser encontram entre 200 e $239 \mathrm{mg} \cdot \mathrm{dL}^{-1}$ serem mais de $25 \%$ ano a ano. O equilibrio corpóreo do colesterol é fundamental para suprir a fisiologia do organismo, uma vez que está esterificado compondo estruturas celulares de membrana, além de participar de metabolismos hepáticos que o direcionam para síntese de hormônios e sais biliares. O consumo de carnes, frutos do mar, ovos, leite cru, derivados lácteos e manteigas como fontes primárias de colesterol devem ser devidamente controlados, porque o corpo possui também a capacidade de síntese $(20 \mathrm{mg}$ de colesterol $/ \mathrm{kg} / \mathrm{dia})$ e o consumo excessivo pode oferecer risco de aumento nos níveis séricos, fator de risco para doenças cardiovasculares ${ }^{[6,21,22]}$.

Ainda sobre o colesterol, porém observando a fração HDL, os valores considerados desejáveis representam valores menores que $15 \%$ a cada ano. Noutro ponto, os valores baixos somam aproximadamente mais que o dobro dos valores desejáveis. Se considerarmos que o restante dos exames observados (aproximados 60\%) está num intervalo transitório, podem-se haver maiores chances de evoluírem para baixos e não desejáveis. Desta forma é evidente a necessidade de estímulo à mudança de hábitos alimentares e estilo de vida uma vez que o colesterol fração HDL médio dos cinco anos estudados é de 46,69 $\pm 2,25 \mathrm{mg} \cdot \mathrm{dL}^{-1}(\mathrm{CV}=$ $4,82 \%$ ) (Dados não apresentados), ou seja, ainda longe da classe desejável. Todo colesterol extra corpóreo provém da dieta deixando a maioria da população inadequada sobre este parâmetro. Fica evidente uma situação onde deveria haver um ajuste de ingestão de gorduras saturadas e trans, privilegiando fontes poli- e monoinsaturadas como alimentos ricos em ácidos graxos ômega 3 e 9,tais quais peixes de água fria (salmão, truta, sardinha, atum), castanhas dessalgadas, azeite de oliva extra virgem e óleo de canola. Estas gorduras conhecidas popularmente como boas, permitem aumento do índice sérico de HDL atuantes na remoção do colesterol dos tecidos periféricos e de outras lipoproteínas enviando-os ao fígado, o que caracteriza a etapa de transporte reverso do colesterol que permite seu metabolismo e excreção[6,21,22].

Em todos os períodos verificados, há predominância da população na faixa desejável dos triglicerídeos com valores sempre superiores a 58\%. Porém, por se tratar de um parâmetro influenciado pela dieta do paciente e sujeito a flutuações em função do preparo adequado do jejum antes da coleta do sangue para o procedimento, as demais 
classes podem interligar-se de forma bastante dinâmica e por isso é importante que haja estímulo a suas diminuições de forma ampla. Isto pode ser ilustrado pela proximidade das classes limítrofe e alta com médias semelhantes. A média de concentração de triglicerídeos nos cinco anos foi de 162,28 \pm 6,53 mg.dL $\mathrm{dL}^{-1}(\mathrm{CV}=4,02 \%)$ (dados não mostrados), considerado limítrofe. Triglicerídeos representam mais de $90 \%$ da gordura da alimentação e $90 \%$ da gordura armazenada nos tecidos, sobretudo adiposo e muscular o que torna esses nutrientes bastante importantes nos processos de metabolismo energético do organismo. Níveis elevados de triglicerídeos estão associados a baixos níveis de colesterol HDL e altos níveis de colesterol LDL. Entre as diversas causas dos níveis elevados estão: inatividade física, excesso de peso ou obesidade, fumo, consumo excessivo de álcool, diabetes do tipo 2, hipotireoidismo e fatores genéticos, todos associados ao aumento do risco de doença cardíaca ${ }^{[15,21-23]}$.

A população araraquarense mostra prevalência de valores normais para glicemia de jejum com frequência superior a $54 \%$ em todos os anos frente ao número equilibrado de indivíduos tolerantes e já diabéticos, onde as frequências estão próximas a 20\%. A glicemia de jejum é o exame mais requisitado nos prontuários médicos, uma vez que a glicose representa o principal componente da dieta responsável por fornecer energia ao metabolismo celular e sua alteração é responsável pelo diabetes sabidamente incurável. Doença esta que, segundo a SBD representa uma epidemia em curso que se especula alcançar trezentos milhões de pessoas até 2030 . Além disso, sua natureza crônica, a gravidade das complicações e os meios necessários para controlá-las, tornam-na uma doença muito onerosa para o paciente e para o sistema de saúde como um todo. No Brasil, os gastos oscilam em US $\$ 3,9$ bi anuais ${ }^{[16]}$. A média da glicemia de jejum é de 111,22 \pm 4,29 mg.dL d $^{-1}(\mathrm{CV}=3,86 \%$ ) (Dados não apresentados ilustrando um quadro de tolerância diminuída a glicose, ou seja, uma população prédiabética). Deve-se ressaltar que outros procedimentos são necessários ao diagnóstico completo de diabetes como o teste oral de tolerância a glicose, estudo de hemoglobina glicada, curva insulínica e marcadores moleculares ${ }^{[16]}$. Estes procedimentos são importantes especialmente na classe de indivíduos identificados como tolerantes. Um dado isolado de glicemia é apenas a primeira etapa de uma investigação criteriosa que parte do preparo de jejum para coleta de sangue periférico até histórico familiar, por exemplo. Assim, não se recomenda observância isolada em nenhum quadro clínico. Outro ponto observado é a ausência da categoria dos indivíduos hipoglicêmicos. São considerados hipoglicêmicos aqueles indivíduos cuja glicemia de jejum apresente valor inferior a 60 mg.dL-116.

Dentre outros itens importantes nas dietas, os minerais também devem ser consumidos de forma equilibrada e o acesso ao mesmo, porvia fontes alimentares saudáveis. Muitos minerais são cofatores enzimáticos e estruturais que auxiliam na homeostase. Um dos elementos recorrentes na clínica médica é o ferro sérico relacionado diretamente com a anemia ferropriva e o cálcio envolvido na formação óssea, coagulação sanguínea, contração muscular e transmissão nervosa. De forma análoga, outro elemento de atenção à saúde coletiva é o sódio que participar ativamente nos equilíbrios osmóticos capazes de se alterarem nos quadros de patologia cardíaca e renal[18,20,23,24].

A população de Araraquara mostra equilibrio para o atributo potássio, porém oscilações maiores para sódio. Na Figura 1, pode-se verificar a distribuição das médias mensais ao longo dos anos selecionados neste trabalho. 


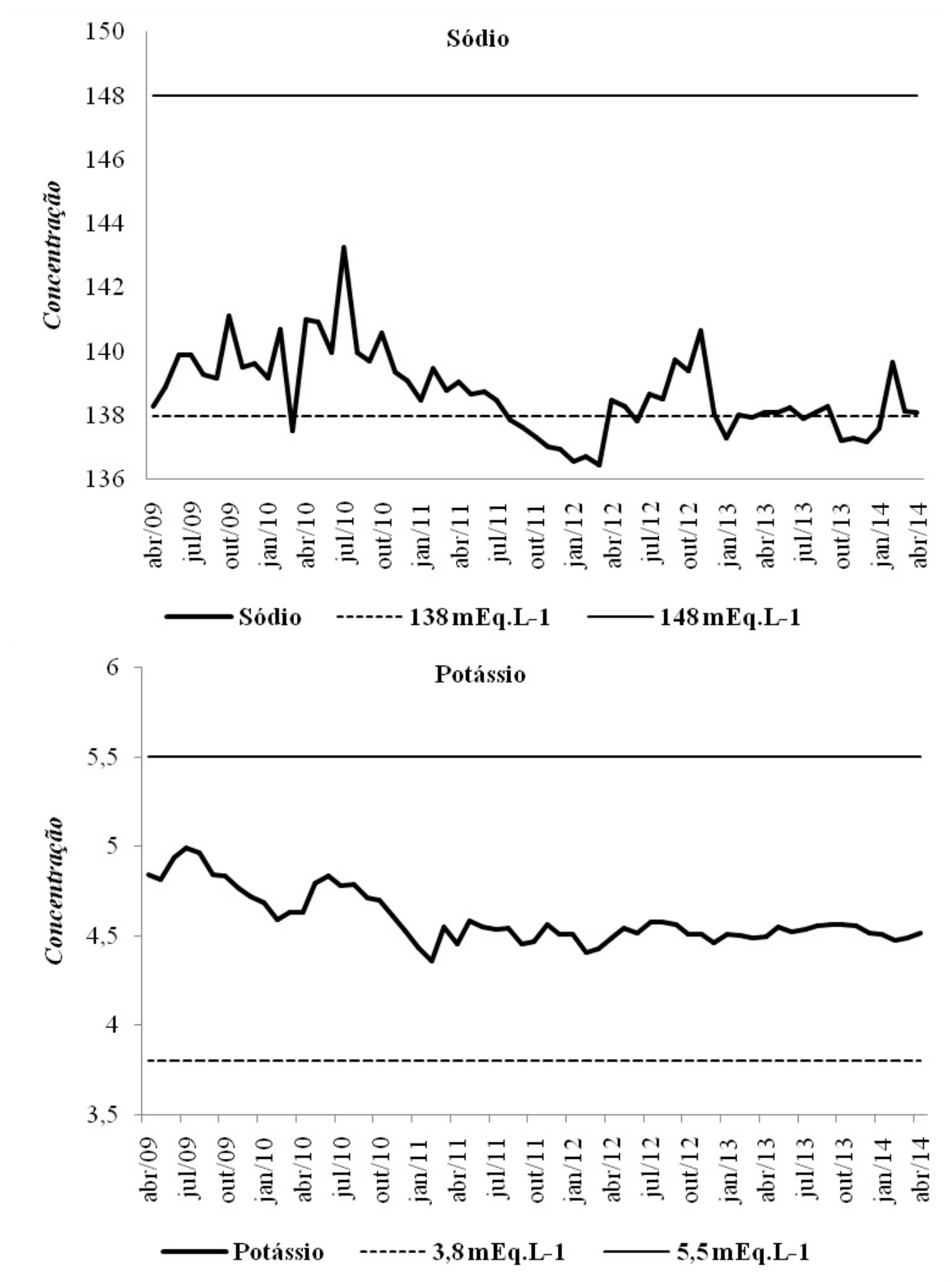

Figura 1: Distribuição de médias dos valores séricos de sódio e potássio entre abril/2009 e abril/2014 obtidos do levantamento realizado no laboratório de análises clínicas da Faculdade de Ciências Farmacêuticas de Araraquara.

Fonte: Autoria própria.

Maior parte do potássio inserido na dieta provém de alimentos básicos e de fácil acesso, além de ser menos expressivo nos alimentos industrializados. Assim, o consumo regular de frutos cítricos, legumes amarelos ou verdes e grãos são fontes comuns aos consumidores. As médias séricas de sódio são mais instáveis mostrando um decréscimo no segundo semestre de 2010 até março de 2012, a partir do qual sofrem discreto aumento até outubro daquele ano. Em 2013, retomam valores menores ficando próximos à referência inferior até final do período analisado. 
As médias observadas nos 61 meses são $138,72 \pm 1,29 \mathrm{mEq} \cdot \mathrm{L}^{-1}(\mathrm{CV}=0,93 \%)$ para sódio e $4,59 \pm 0,15(\mathrm{n}=89760, \mathrm{CV}=3,27 \%) \mathrm{mEq} \cdot \mathrm{L}^{-1}$ para potássio séricos. Estes eletrólitos são muito influenciados pelo nível de hidratação do indivíduo e sofrem oscilações em quadros de diarreia e vômitos, muito comuns também na rotina clínica e uma queixa bastante frequente durante o atendimento médico. O fato de poder acometer, sobretudo crianças e, portanto relacionar-se com a mortalidade infantil, são itens de alerta em saúde pública. Segundo a Organização Mundial de Saúde (OMS), o consumo regular destes deve ser de menos de 2,0g de sódio e pelo menos $3,51 \mathrm{~g}$ de potássio o que equivale a menos de uma colher de chá rasa de sal de cozinha. Dietas vegetarianas podem ocasionar discreta redução na pressão arterial sistólica em hipertensos leves. O estilo de vida vegetariano com atividade física regular, controle de peso, aumento do consumo de potássio e baixa ingestão de álcool e a dieta em si, rica em fibras, pode ser favorável na redução do risco cardiovascular. Devem integras a dieta alimentos pobres em sódio e ricos em potássio, como feijões, ervilha, vegetais de cor verde-escuro, banana, melão, cenoura, beterraba, frutas secas, tomate, batata inglesa e laranja ${ }^{[18-20,23,24]}$. A dieta com o consumo de frutas, verduras, cereais integrais, leite desnatado e seus derivados, quantidade reduzida de gorduras saturadas e colesterol, maior quantidade de fibras, potássio, cálcio e magnésio, associada à redução no consumo de sal é fortemente recomendada especialmente para hipertensos ${ }^{[18,20,23]}$. Desta forma, o controle do consumo do sal iodado, ou popularmente, o sal de cozinha funciona como uma ferramenta para auxiliar o controle de alterações metabólicas. Este alimento tem sido considerado importante fator no desenvolvimento e na intensidade da hipertensão arterial. $\mathrm{O}$ excesso do sódio eleva a pressão arterial por aumento de volemia e aumento do débito cardíaco. Em seguida, por auto-regulação, há aumento da resistência vascular periférica, que mantem elevada a pressão arterial. A ingestão excessiva de sal ativa diversos mecanismos pressores: como aumento de vasoconstrição renal, reatividade vascular aos agentes vasoconstritores e elevação de inibidores das enzimas da bomba sódio-potássio. Por isso, a orientação nutricional foca na redução de embutidos, enlatados, defumados, bebidas isotônicas, caldos em tabletes e molhos prontos ${ }^{[15,23,24]}$.

Como os eletrólitos sanguíneos são importantes na movimentação das soluções pelos compartimentos corpóreos, devido aos seus efeitos osmóticos, ajudam na manutenção do equilíbrio ácido-base, são ativadores enzimáticos ou coenzimas, agem de forma contrária e complementar como na propagação dos impulsos nervosos ou na relaxação do sistema nervoso central. Seu controle deve ser tal que atuem harmônicos e equilibradamente no organismo. $\mathrm{O}$ sódio, em particular, está relacionado ao transporte de dióxido de carbono no metabolismo do bicarbonato, provoca irritabilidade nervosa e muscular funcionando como marca-passo do coração, além de ser indispensável na absorção de glicose e transporte de nutrientes no intestino. O potássio está relacionado ao transporte de oxigênio e gás carbônico nas hemácias, controla viscosidade plasmática, auxilia o metabolismo hepático do glicogênio e na oxirredução da glicose. Tal distribuição funcional mostra versatilidade dos equilíbrios onde estão envolvidos e os quadros patológicos onde se inserem demostram a importância de manutenção osmótica do organismo que pode refletir na pressão arterial e função renal, situação indesejada e agravante de histórico diabético, sobrepeso e disfunção hormonal[19].

Entretanto, alimentar-se não é uma tarefa simples e o controle de ingestão pode representar grande problema para o indivíduo seja pela dificuldade motora propriamente dita ou por repetitivo ou desestimulante. Isto tudo pode tornar ainda mais delicado para idosos, crianças ou pacientes imuno suprimidos. Desta maneira, a abordagem terapêutica das Doenças Crônicas Não Transmissíveis passa a contar com auxílio de medicamentos capazes de complementar o tratamento diário (dietas, caminhadas, exercícios resistivos, entre outros) ou mesmo suplantar motivos genéticos como uma produção aumentada de colesterol ou insulina, por exemplo. Como esta possibilidade de tratamento, é para muitos, única, diversos medicamentos são lançados e estudados ampliando a gama de terapias. Tal fato complementa o quadro do aumento de gastos com medicamentos e monitoramentos pela esfera pública, uma vez que há uma dependência concreta dos mesmos para qualidade de sobrevida do paciente. Neste contexto existe a figura das Farmácias Populares que atingem principalmente às pessoas que interrompem o tratamento por não conseguirem arcar com o preço dos medicamentos contínuos. Hoje, o programa conta com 97 medicamentos essenciais para hipertensão, diabetes, úlceras gástricas, asma, depressão e verminoses, além inclusive de preservativos e anticoncepcionais. Com isso, há uma maior chance de continuidade das terapias 
resultando em uma melhoria da saúde dos indivíduos com alguma carência social ou financeira. Em Araraquara, dentre os medicamentos mais aplicados e distribuídos por intermédio das Farmácias Populares estão os indicados para controle de hipertensão arterial, diuréticos, tratamento de dislipidemias e auxiliares do metabolismo de glicose. Entre outros: ácido acetilsalicílico, atenolol, captopril, digoxina, (di)nitrato de isossorbida, enalapril, furosemida, glibenclamida, hidroclorotiazida, losartana, metformina, nifedipina, propranolol, sinvastatina e verapamina $^{[25]}$.

\section{CONCLUSÃO}

Há uma população com tendência a quadros de risco clínico para diabetes e dislipidemia frente à melhoria de rendimentos e promoção socioeconômica. Araraquara revelou $77 \%$ de sua população com vulnerabilidade baixa e muito baixa juntas com rendimentos médios de $\mathrm{R} \$ 2564,00$ entre 2008 e 2010. Sobre os rendimentos dos empregados formais observou-se um acúmulo médio positivo em todas as classes: número de empregos formais $(25 \%)$; setores agropecuários, silvícolas e pescado $(45 \%)$; indústria (31\%); construção einfraestrutura (44\%); serviços e veículos (27\%) entre 2009 e 2012. Além disso, mostrou prosperidade industrial, econômica, social e sanitária: baixa mortalidade $(14,14$ por mil), alta alfabetização $(94,80 \%)$, alto IDH $(0,815)$, renda per capita acima do mínimo (R \$ 891,74), amplo abastecimento de água (99,43\%), coleta de lixo $(99,96 \%)$ e esgotamento sanitário $(98,89 \%)$ em 2010; porém a atenção à saúde ainda demanda cifras crescentes o que a médio e longo prazo, parece ser um problema grave visto $\mathrm{O}$ aumento da expectativa de vida (72,17 anos em 2010) e provável maior número de futuras Doenças Crônicas Não Transmissíveis. Os gastos em saúde também mostram acúmulo médio positivo: total de despesas (20\%); assistência hospitalar (24\%); vigilância sanitária $(18 \%)$ e epidemiológica (41\%) entre 2009 e 2011; unidades de atenção básica (10\%); leitos de internação (8\%) e SUS (13\%) entre 2009 e 2013. Ainda que haja um aumento do potencial socioeconômico que reflete também num aumento per capita de calorias, e em tese uma alimentação de melhor qualidade, as gorduras e açúcares parecem mais frequentes que cereais integrais, leguminosas, frutas e vegetais. Este aspecto, dito de transição parece ruim (e moderno), afinal o alimento tradicional e in natura passa a ser processado aumentando a densidade energética e diminuindo o aspecto nutricional. Resultado disso tem-se a predisposição ao diabetes, aumento de risco cardiovascular e obesidade como foi observado nas maiores frequências em níveis intermediários dos parâmetros glicemia de jejum e HDL, junto a frequências semelhantes em níveis de risco mais grave como nos dados de triglicerídeos, que ilustram essa postura delicada entre estados saudáveis e patologias em potencial. Desta maneira, a Segurança Alimentar e Nutricional é um tema articulado àsaúde, ao ambiente e ao desenvolvimento sustentável global e passa por novas estratégias de educação alimentar e nutricional, estímulo da manutenção de massa corporal adequada, melhor capacitação dos profissionais da saúde clínica e nutricional, melhoria a atenção dos transtornos alimentares, política econômica igualitária e promoção da qualidade de vida. Por isso, as interrelações epidemiológicas observadas são importantes instrumentos para gerar e comprovar hipóteses sobre a relação alimentação-doença sendo necessárias mais pesquisa a fim de evidenciar como um fator alimentar determina um efeito na saúde do indivíduo ou de uma população.

\section{REFERÊNCIAS}

[1] Marchioni DML, Fisberg RM. Dieta, nutrição e prevenção de doenças crônicas não transmissíveis. In: Cuppari L organizador Nutrição nas doenças crônicas não-transmissíveis. São Paulo: Manole; 2009. p. 1-25.

[2] Oliveira, MR, Fontes, GG, Lima, LM, et al. Avaliação bioquímica do sangue. In: Calixto-Lima, L, Reis, NT organizadores. Interpretação de exames laboratoriais aplicados à nutrição clínica. Rio de Janeiro: Rubrio; 2012. p. 17-38.

[3] Valente FLS. Segurança alimentar e nutricional: transformando natureza em gente [Internet]. Juiz de Fora: COMSEA; 2003 [Acesso em: 28 nov 2013]. Disponível em:

http://www.pjf.mg.gov.br/sg/conselhos/seguranca_alime ntar/documentos/natureza_gente.pdf. Sem paginação.

[4] Maluf, RS, Menezes, F, Valente, FLS. Contribuição ao tema de segurança alimentar no Brasil. Cad Debate. 1996;4:66-88.

[5] Valente, FLS. O combate a fome e a desnutrição e a promoção da alimentação adequada no contexto do direito humano a alimentação: um eixo estratégico do desenvolvimento humano sustentável [Internet]; 2001, mimeo, apud. Belik, W, da Silva, JG, Takagi, M. Políticas de combate à fome no Brasil. São Paulo Perspec. [Internet]. 2001. [Acesso em 11 dez 2015]. 15 (4), 119$129 . \quad$ Disponível em: http://www.scielo.br/scielo.php?script=sci_arttext\&pid= S0102-88392001000400013\&lng=pt\&nrm =iso\&tlng=pt. 
[6 Paula, ID, Machado, JC. Dislipidemias. In: Vannucchi, H, Marchini, JS organizadores. Nutrição e metabolismo: nutrição clínica. Rio de Janeiro: Guanabara Koogan; 2007. p.205-220.

[7] Council for International Organizations of Medical Sciences. International ethical guidelines for biomedical research involving humans subjects. Geneva: WHO; 1993. $63 \mathrm{p}$.

[8] IBGE: Instituto Brasileiro de Geografia e Estatística [Internet]. Brasília: IBGE; c2014. Cidades: Araraquara; [Acesso em: 09 ago 2014]; [cerca de 2 telas]. Disponível em: <http://cod.ibge.gov.br/233P9>.

[9] IMP: Informação dos Municípios Paulistas [Internet]. São Paulo: SEADE. Perfil municipal: Araraquara; [Acesso em: 09 ago 2014]; [cerca de 6 telas]. Disponível em:

$<$ http://www.imp.seade.gov.br/frontend/\#/>.

[10] Departamento Autônomo de Água e Esgotos. Plano municipal de saneamento básico.: relatório de minuta do plano consolidado. Araraquara: Prefeitura Municipal de Araraquara, 2014. 376 p. Disponível em:

<http://www.daaeararaquara.com.br/PMSB/PMSB-

RELATORIO $\% 20$ DE $\% 20$ MINUTA $\% 20$ DO $\% 20$ PLAN O\%20CONSOLIDADO.pdf.>.

[11] Assembléia Legislativa do Estado de São Paulo: $18^{\mathrm{a}}$ legislatura. São Paulo: SEADE. Indice paulista de responsabilidade social e de vulnerabilidade social: Araraquara; [Acesso em: 09 ago 2014]; [cerca de 2 telas]. Disponível em: <http://indices-ilp.al.sp.gov.br/>.

[12] Villar ES. Epidemiologia nutricional: uma nova disciplina. In: Oliveira JED, Marchini JS organizadores. Ciências nutricionais: aprendendo a aprender. 2 ed. São Paulo: Sarvier; 2008. p. 601-609.

[13] Cassan, RSL, Schimdt A, Rabito EI, et al. Avaliação antropométrica e estado nutricional. In: Oliveira, JED, Marchini JS organizadores. Ciências nutricionais: aprendendo a aprender. 2 ed. São Paulo: Sarvier; 2008. p. 613-635.

[14] Mais de 110 mil araraquarenses são usuários de plano de saúde [Internet]. Araraquara Tribuna [Internet]. 01 mar 2014 [Acesso em 14 ago 2014]; Noticias: [cerca de 3 p.] Disponível em: http://www.araraquara.com/noticias/noticias_internaN OT.aspx?idnoticia=929441.

[15] Xavier HT, Izar MC, Faria Neto JR, et al. V diretriz brasileira de dislipidemias e prevenção da aterosclerose. Arq Bras Cardiol; 2013; 101, Supl 1(4): 1-22. Disponível em: http://www.scielo.br/scielo.php?pid=S0066-

782X2013004100001\&script=sci_arttext.
[10] Oliveira JEP, Venâncio S organizadores. Diretrizes da Sociedade Brasileira de Diabetes 2013 - 2014. São Paulo: AC Farmacêutica; 2014. 365 p. Disponível em: http://www.nutritotal.com.br/diretrizes/files/342-diretrizessbd.pdf.

[17] Rennke HG, Denker BM. Fisiopatologia renal: princípios básicos. São Paulo: Livraria Médica Paulista; 2009. 394 p.

[18] Bazanelli AP, Baria P, Lopes MGG. Doença renal crônica. In: Calixto-Lima L, Reis NT organizadores. Interpretação de exames laboratoriais aplicados à nutrição clínica. Rio de Janeiro: Rubrio; 2012. p. 327-343.

[19] Pedroso, ERP. Água e eletrólitos. In: Oliveira JED, Marchini JS organizadores. Ciências nutricionais: aprendendo a aprender. 2 ed. São Paulo: Sarvier; 2008. p. 613-635.

[20] Wiegert EVM, Calixto-Lima L, Costa NMB. Minerais. In: Calixto-Lima L, Reis NT organizadores. Interpretação de exames laboratoriais aplicados à nutrição clínica. Rio de Janeiro: Rubrio; 2012. p. 137-152.

[21] Lottemberg AMP, Buonacorso V. Dislipidemias. In: Cuppari L organizador. Nutrição nas Doenças Crônicas Não Transmissíveis. São Paulo: Manole; 2009. p.191-217.

22] Calixto-Lima L, Guedes EP, Reis NT. Dislipidemia. In: Calixto-Lima, L, Reis, NT organizadores. Interpretação de exames laboratoriais aplicados à nutrição clínica. Rio de Janeiro: Rubrio; 2012. p. 213-226

[23] Costa RP, Mendonça LT. Doenças cardiovasculares. In: Cuppari L organizadores. Nutrição nas Doenças Crônicas Não Transmissíveis. São Paulo: Manole; 2009. p. 219-265.

[24] Navarro AM, Oliveira LA, Ferreira JF, et al. Excreção urinária de sódio em crianças e adolescentes de escolas urbanas e rurais. Alim Nutr Araraquara. 2009; 20(4): $657-$ 661. Disponível em: http://servbib.fcfar.unesp.br/seer/index.php/alimentos/article/view Article/1260

[25] Brasil. Ministério da Saúde. Portal de Saúde. Farmácia Popular do Brasil. Lista de medicamentos disponibilizados pelo "Aqui tem farmácia popular" [internet]; 2014 [Acesso em 04 nov 2014]. Disponível em: <http://portalsaude.saude.gov.br> 